\title{
Closer look at the flight dynamics of wings with non-elliptic lift distributions
}

\author{
Estela Bragado Aldana*, Arthur Mendez $z^{\dagger}$ and Mudassir Lone \\ Cranfield University, Cranfield, MK43 OAL, United Kingdom
}

\begin{abstract}
Prandtl's alternative solution for wings with minimum induced drag opens another route for improving aircraft performance especially at the conceptual design phase. In this work, the lateral-directional characteristics of such wings are studied for a glider use case from a perspective that focuses on proverse yaw and handling qualities. The authors propose an aileron design methodology that ensures roll authority and proverse yaw characteristics. The results show that these conditions cannot be satisfied by a conventional Elliptic configuration, whereas wings with non-elliptic distributions provide several solutions. Non-linear flight dynamic models of the configurations that provided maximum proverse yaw were assessed in an engineering simulator and compared against the baseline Elliptic case. Proverse yaw was observed in the simulation data and pilot feedback indicated improved handling qualities in the non-elliptic cases. However, the inherent directional instability combined with other lateral-directional coupling effects masked the observations from the pilot's perspective.
\end{abstract}

\section{Introduction}

$\mathrm{T}$ HE challenge faced by aeronautical engineers today is how to extract every gram of efficiency from the design being considered. Quite rightly, majority of attention is focused on the aircraft in its cruise conditions where it spends most of its operational time. Scenarios where the aircraft is manoeuvring and increases in drag are simply accepted are treated as a nuisance[1]. During a lateral turn, for instance, adverse yaw manifests due to differential drag caused by aileron deflection and is usually solved with the use of additional drag with the help of flight control systems, making the manoeuvre an aerodynamically inefficient exercise. Nevertheless, bird flight demonstrates that manoeuvring flight is possible without the requirement of additional drag or a vertical stabilizer. In this paper, the authors explore the applicability of wing designs based on Prandtl's[2] proposed non-elliptic lift distributions that seem to promise not only more efficient cruise but also drag optimal coordinated turns. In Reference 2, Prandtl presents an alternative solution for minimum induced drag that, unlike the Lifting Line theory [3], brings together aerodynamic and structural design requirements by allowing the span to extend. Altogether, the process results in a bell-shaped curve as the optimal spanload, achieved through a non-linear wing twist distribution. This effectively reduces the wing-root bending moment and creates negative induced drag on the outboard wing. Here, the drag differential during a turn becomes beneficial when the induced thrust is manipulated accordingly by an aileron designed for this purpose [4-6]. However, because of the distinctive lightly loaded wingtips, the design could lead to insufficient roll authority, and therefore it requires reconsideration of the aileron sizing parameters. The Horten brothers[7] independently explored this solution on many of their tailless aircraft models, achieving successful flights without vertical control surfaces. Recently, Bowers[8] has observed the phenomenon of proverse yaw in numerous flight tests using NASA's PRANDTL-D flying wing.

The aim of this work is to assess the flight dynamic characteristics of wings with non-elliptic lift distributions, focusing on proverse yaw and the implications on aileron design. The work presents an analytical methodology based on reduced order methods to size the ailerons for maximising proverse yaw while providing the required roll authority. Under these objectives, the assessment of the lateral-directional characteristics and handling qualities of three different sailplane configurations are presented and compared. The evaluation of handling qualities uses an engineering flight simulator and non-linear flight dynamic models. Cranfield University's Future Systems Simulator (FSS) has been used for the integration of the dynamic models and a flight testing framework, consisting on two different turning manoeuvres designed for the investigation of lateral-directional handling qualities. The initial analytical lateral-directional handling qualities study has been compared to the data collected from pilot-in-the-loop simulations.

\footnotetext{
*PhD Researcher, Dynamics, Simulation and Control Group, Cranfield University, AIAA student member.

${ }^{\dagger}$ Research Assistant in Flight Control, Dynamics, Simulation and Control Group, Cranfield University.

${ }^{\ddagger}$ Senior Lecturer in Flight Dynamics, Dynamics, Simulation and Control Group, Cranfield University, AIAA Senior member.
} 


\section{Case study}

The baseline model chosen for this study is the Silent 2 Targa Pure[9] sailplane, shown in Figure[1] This low-speed, high performance, high aspect-ratio glider designed with a crescent planform provides a suitable initial design point to which Prandtl's 1933 theory can be applied. The geometries of the two equivalent wings with non-elliptic spanloads have been derived using Lifting Line theory combined with Prandtl's 1933 assumptions for minimum induced drag and structural equivalence[10]. Two different non-elliptic circulation distributions were defined to obtain each of the planforms and twist distributions: The first planform is obtained using a trigonometric function limited to the first term of the cosine Fourier series and the second planform is obtain via Prandtl's 1933 optimal spanload solution. More detail on the derivation of these configurations can be found in Reference 10. With this, the validity of this comparative methodology relies on the definition of an equivalent wing. In this work, aerodynamic and structural equivalence have been assumed through a set of design constraints that satisfy Prandtl's 1933 assumptions. Following these considerations, the main differences between the three sailplane models will come from the planform design.

For analytical simplicity and to obtain the traditional elliptic distribution for minimum induced drag the baseline planform has been modelled as an ellipse. Other characteristics will be equal for all configurations. For instance, the wing area, the design flight conditions and the mass, which is also unchanged due to the assumption of structural equivalence. The fin and rudder configuration also remain equal for the three models even though this stabiliser is usually designed concurrently with the aileron to achieve the required coupling of the lateral-directional characteristics. This has been done to nominalise the contribution from aerodynamic surface and highlight the differences due to the wings. The main design parameters are summarised in Table 1 .

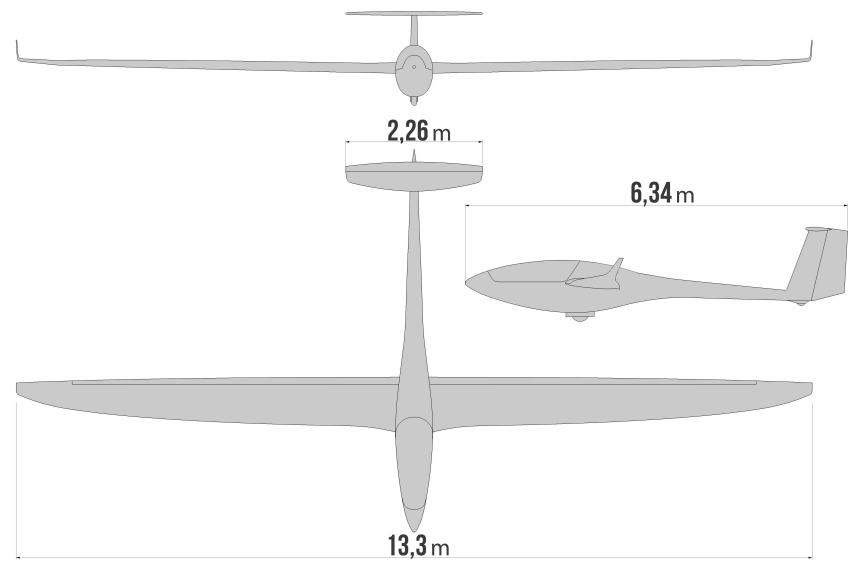

Fig. 1 Silent 2 Targa Pure layout[ $[9]$.

Table 1 Reference geometric characteristics and design flight conditions of the models.

\begin{tabular}{|l|c|c|c|}
\hline \hline Configuration & Elliptic & Cosine & Prandtl \\
\hline \hline Span, $b(m)$ & 13.3 & 18.4 & 16.3 \\
\hline Aspect ratio, $A R$ & 20 & 38 & 29 \\
\hline Root chord, $c_{\text {root }}(m)$ & 0.85 & 0.89 & 1.00 \\
\hline Taper ratio, $\lambda$ & \multicolumn{3}{|c|}{0.1} \\
\hline Ref. wing area, $S_{\text {ref }}\left(m^{2}\right)$ & \multicolumn{3}{|c|}{300} \\
\hline Mass, $m(k g)$ & \multicolumn{3}{|c|}{0.913} \\
\hline Lift coefficient, $C_{L}$ & \multicolumn{3}{|c|}{23012} \\
\hline Airfoil $(\mathrm{NACA})$ & 25 \\
\hline Speed, $V(m / s)$ & 600 \\
\hline Altitude, $h(m)$ & \multicolumn{3}{|c|}{} \\
\hline \hline
\end{tabular}




\section{Aileron design methodology and optimisation}

The methodology developed in this work aims to derive a design criteria for an aileron geometry that provides roll authority and nullifies adverse yaw for a wing with non-elliptic lift distribution. The proposed problem is addressed through the assessment of the lateral-directional derivatives relevant in the design requirements for roll control and its secondary effects. Following these conditions, the output of the problem will be the aileron chord ratio and its inboard and outboard spanwise sections that satisfy both given objectives.

\section{A. Problem definition}

The design criteria chosen to size the ailerons for roll effectiveness and proverse yaw focuses on achieving two goals. Firstly achieving the minimum required roll authority, which is determined by certification specification[11] and is given by the static characteristics of the wing during aileron deflection in terms of a prescribed non-dimensional roll-rate. Secondly obtaining the maximum proverse yaw qualitatively, where following the standard right-hand stability axes, the yawing moment due to aileron deflection will be negative. This problem has been formulated analytically to provide some insight into the consequences of deflecting an aileron that is placed on a region of induced thrust. Aileron deflection has been accounted for via two-dimensional plain flap theory. Lifting line theory has been subsequently used to derive the three-dimensional wing properties, combined with strip theory to obtain expressions for stability derivatives. The reason for this stems from the need to avoid the use of empirical approximations or other methods that will bypass the local effects on the induced drag distribution, since traditional methods tend to be based on the elliptic solution as the optimal for minimum induced drag[12-14]. At this stage, only the contributions from the wing have been considered.

\section{B. Theoretical background and derivation}

The primary function of an aileron is the lateral-directional control of an aircraft, which is governed primarily through roll rate $p$ and the consequent changes in heading. Lateral control research is mainly concerned with the design of control devices that provide enough effectiveness at all airspeeds within the flight envelope, as well as the ability to produce a required roll rate. Therefore, the requirements for satisfactory lateral control must be carefully specified. Among the various criteria proposed for specifying roll performance, analytical studies and pilot experience have indicated that the design point based on the non-dimensional roll rate $p b / 2 \mathrm{~V}$, or helix angle, is most convenient to specify roll authority[15-[18]. This parameter is independent of altitude for given aileron deflections and is also independent of aircraft mass properties for geometrically similar aeroplanes. From a design perspective, this means that the aileron size can be determined by the required steady state roll rate. In the case of subsonic aircraft such responsiveness must be assured at slow speeds with large deflections and will be determined by design requirements and certification specifications.

From the one degree-of-freedom equations of motion of a rigid aircraft, where it is assumed that the roll rate $p$ is damped due to the local variation in angle-of-attack. The rolling motion caused by a given mean aileron deflection $\delta_{a}$ can be expressed as [19, 20]:

$$
\frac{p b}{2 V}=-\frac{C_{l_{\delta_{a}}}}{C_{l_{p}}} \delta_{a}
$$

where $C_{l_{\delta_{a}}}$ is the rolling moment coefficient due to aileron deflection and $C_{l_{p}}$ is the rolling moment coefficient due to roll rate. The conditions to determine the required roll performance are specified in this case in CS-22[11], where the aircraft must be able to reverse the direction of a turn with a $45^{\circ}$ bank in the opposite direction within $b / 3$ seconds, at a speed of 1.4 times the stalling speed $V_{S 1}$, and with maximum deflection $\delta_{a_{\max }}$ allowed by the aileron[21]. The estimation the roll control derivative can be done by applying strip theory and assuming that the controller is a simple flap-like device, such that[22]:

$$
C_{l_{\delta_{a}}}=-\frac{2}{S b} \int_{y r_{y 1}}^{y r_{y 2}} C_{l_{f}} c(y) y d y
$$

where $c(y)$ is the wing chord at a given spanwise location $y$, for which the integration limits are defined by the aileron inboard and outboard sections, designated by the ratios $r_{y 1}$ and $r_{y 2}$ respectively, over the semi-span. The lift coefficient due to trailing edge control surface deflection $C_{l_{f}}$ has been calculated through flat plate theory for a flap device. Considering a symmetric aerofoil and assuming a chordwise distribution of vorticity in the form of a series, the 
two-dimensional flap lift effectiveness can be computed as [23, 24]:

$$
\frac{C_{l_{f}}}{C_{l_{\alpha}}}=k_{3 D} \frac{2}{\pi}\left[\sin ^{-1} \sqrt{\frac{c_{f}}{c}}+\sqrt{\frac{c_{f}}{c}\left(1-\frac{c_{f}}{c}\right)}\right]
$$

where $C_{l_{\alpha}}$ is the lift curve slope, which is constant in this case since a single aerofoil distribution has been used. The factor $k_{3 D}$ considers the losses due to three-dimensional effects, and $c_{f}$ is the chord of the trailing edge control surface. The small perturbation approach used in this methodology is followed by the assumption that $C_{l_{f}}$ remains in the linear range, which agrees with the resulting aerodynamic properties of ailerons observed from experimental data[25].

The roll damping derivative is also calculated with strip theory and integrated along the wing span, yielding[22]:

$$
C_{l_{p}}=-\frac{1}{S_{\text {ref }} b^{2}} \int_{-b / 2}^{b / 2}\left(C_{l_{\alpha}}+C_{d}(y)\right) c(y) y^{2} d y
$$

where $C_{d}(y)$ is the spanwise drag distribution, which sees a spanwise perturbation in incidence angle-of-attack $\alpha \cong p y / V$ due to the roll rate.

Nonetheless, as mentioned earlier, one of the main concerns of this study is the secondary effects of lateral control due to aileron deflection, which will effect directional motion in the form of adverse yaw. This phenomenon can be described as the opposing moment caused by variation of the drag with aileron deflection. Estimates for this derivative are hard to predict and are usually obtained from empirical data or ESDU data items. However, such methods are based on the elliptic spanload as the ideal solution for minimum drag, which therefore overrules the unconventional behaviour of the drag in non-elliptic lift distributions, which will be discussed later. Applying strip theory and assuming small perturbations this can be captured as[22]:

$$
C_{n_{\delta_{a} \text { wing }}}=\frac{1}{S_{\text {ref }} b} \int_{-b / 2}^{b / 2} \frac{\partial C_{d}(y)}{\partial \delta_{a}} c(y) y d y
$$

The following assumptions were made to obtain $\partial C_{d}(y) / \partial \delta_{a}$, where the drag varies with both profile and induced drag as:

$$
C_{d}(y)=C_{d_{0}}(y)+C_{d_{i}}(y)
$$

where the profile drag variation is usually negligible[14]. The induced drag is generally expressed by [26, 27]

$$
C_{d_{i}}(y)=C_{l}(y) \alpha_{i}(y)
$$

Therefore, the variation in section drag due to aileron deflection becomes:

$$
\frac{\partial C_{d}}{\partial \delta_{a}}=C_{l} \frac{\partial \alpha_{i}}{\partial \delta_{a}}+\alpha_{i} C_{l_{f}}+\frac{\partial C_{d_{0}}}{\partial \delta_{a}}
$$

The lift distribution after control surface perturbation can be calculated with the use of Equation 3 as:

$$
C_{l}=C_{l_{0}}+C_{l_{\alpha}} \alpha+\frac{\partial C_{l}(y)}{\partial \delta_{a}} \delta_{a}=C_{l_{(\text {clean })}}+C_{l_{f}} \delta_{a}
$$

To obtain the induced angle of attack $\alpha_{i}$, it is necessary to account for three-dimensional effects, derived from Biot-Savart's formula[27]:

$$
\alpha_{i}(y)=\frac{1}{4 \pi V_{\infty}} \int_{b / 2}^{b / 2} \frac{d \Gamma / d y}{y-y_{0}} d y_{0}
$$

where the circulation distribution $\Gamma(y)$ after aileron deflection can be obtained from the lift coefficient distribution calculated in Equation 9 as:

$$
\Gamma(y)=\frac{C_{l}(y) V_{\infty} c(y)}{2}
$$

Now, it is possible to define the problem analytically where our main objective will be to maximise proverse yaw given the above conditions and obtain the design variables that will determine the aileron geometry, given by: $r_{f}, r_{y_{1}}$ and $r_{y_{2}}$, which remain unknown, and where $r_{f}=c_{f} / c$. Thus, the derivatives due to aileron deflection can be defined as:

$$
C_{l_{\delta_{a}}}=f\left(r_{f}, r_{y_{1}}, r_{y_{2}}\right) \quad \text { and } \quad C_{n_{\delta_{a}}}=f\left(r_{f}, r_{y_{1}}, r_{y_{1}}\right)
$$


From historical data 28-31], we can extract the lower and upper boundaries for the design variables, as well as extract the maximum aileron deflection that will be assigned for this study:

$$
0.15 \leq r_{f} \leq 0.35, \quad 0.5 \leq r_{y_{1}} \leq 0.85, \quad 0.7 \leq r_{y_{2}} \leq 1.0, \quad \delta_{a_{\max }}= \pm 20^{\circ}
$$

In order to ensure that the minimum roll authority required is achieved, the condition that must be satisfied can be expressed as:

$$
\frac{p_{r e q} b}{2.8 V_{S 1}}+\frac{C_{l_{\delta a}}}{C_{l_{p}}} \delta_{a} \geq 0
$$

Proverse yaw is determined by the sign of $C_{n_{\delta_{a}}}$, where it is desired to have a yawing moment in the same direction as the rolling moment. By definition and according to the standard right-hand stability axes[22] $C_{l_{\delta_{a}}}<0$, and thus $C_{n_{\delta a}}$ should be of the same sign:

$$
C_{n_{\delta_{a}}}<0
$$

Out of all the solutions that satisfy the above constraints, the optimal aileron geometry will be the one that yields maximum proverse yaw.

\section{Theoretical results}

The solutions derived from the methodology described above provide insight into the potential of non-elliptic lift distributions for minimising adverse yaw. Figures 2 to 4 show the design space that has been covered, delimited by the upper and lower boundaries of the design variables determined in Equation 13 . Only the solutions that satisfy the conditions from Equations 14 and 15 have been plotted in the searched space. As expected from a conventional wing, the methodology could not find an aileron geometry that would yield proverse yaw for the Elliptic case.

However, various solutions were found for both non-elliptic cases. These results are shifted towards the upper boundaries of the three geometric variables. This is due to both problem constraints: a very low aileron chord ratio $r_{f}$ would not provide enough authority to roll. In the case of the spanwise positions of the aileron, only the geometries found within the region of induced thrust yielded proverse yaw. Comparing the space of solutions of the Cosine and the Prandtl cases, shown in Figures 4 and 3 respectively, it is seen that the range of solutions in the Cosine case is higher, and more solutions are found inboard of the wing. Inspecting the induced drag coefficient distribution of these wings in clean configuration in Figures 6 and 7 , it is seen that the given range of solutions corresponds to their respective regions of induced thrust. In the case of the Cosine distribution, it is found in the outboard wing starting at around 55\% of the semi-span, whereas in the Prandtl distribution the local induced drag becomes negative at approximately $70 \%$ of the semi-span.

Following the problem definition, the aileron geometry that provides the maximum proverse yaw was found, highlighted with a red dot in the design spaces. Figure 8 shows the comparison of the three resultant wings and aileron sizing together with their respective twist distributions. Table 2 summarises the geometric properties of the ailerons and the resultant values for the stability derivatives. It must be noted that the Elliptic configuration remained as the original baseline model, equipped with flaperons, since no solution was found for this configuration.

The variation of induced drag due to aileron deflection can be visualised in Figures ?? to 7, plotted from AVL[32], for a negative bank simulated at various positive aileron deflections. Recalling the definition of adverse yaw, the induced drag along the left wing of the Elliptic configuration plotted in Figure ?? sees a reduction due to the down-going left aileron. In contrast, Figures 6 and 7 show that the induced drag over the left wing of the non-elliptic cases increases with the downwards deflection of the aileron. This increase brings a favourable yawing moment, and overall, a lower total drag per area increase when compared to the elliptic case. 


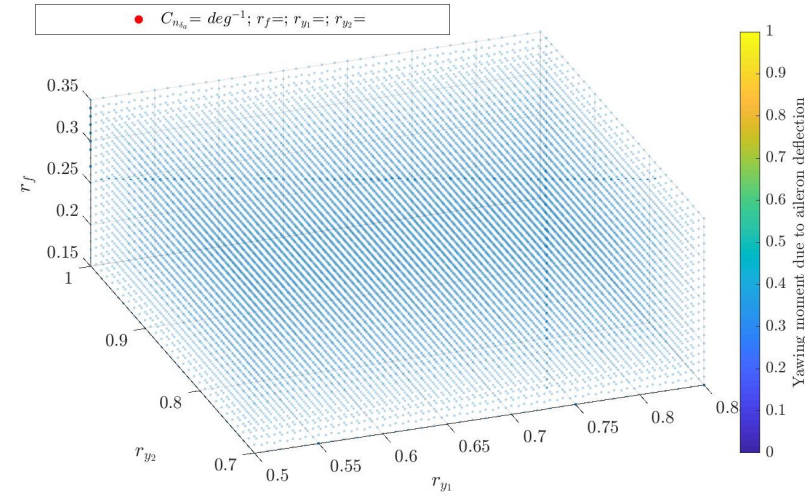

Fig. 2 Design space and solutions that satisfy the problem conditions for the Elliptic case.

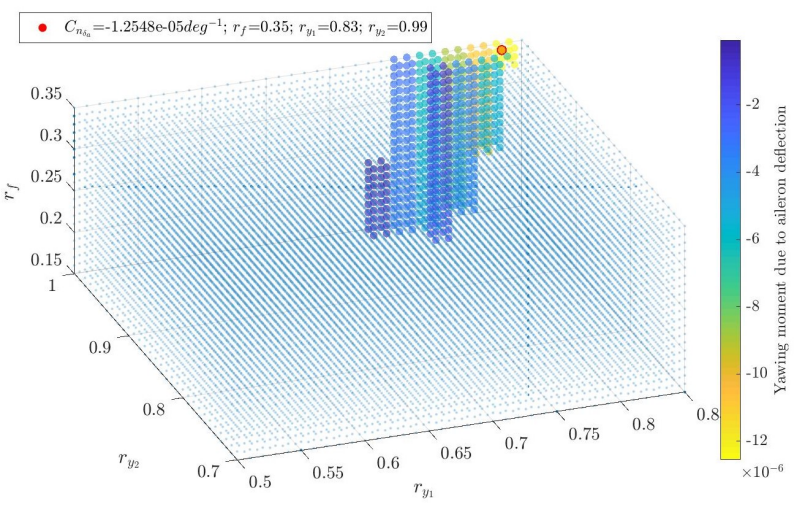

Fig. 3 Design space and solutions that satisfy the problem conditions for the Prandtl case.

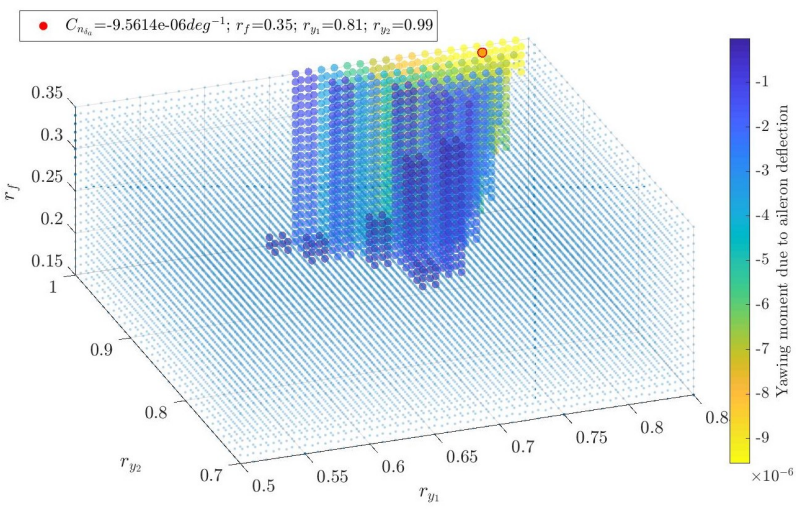

Fig. 4 Design space and solutions that satisfy the problem conditions for the Cosine case.

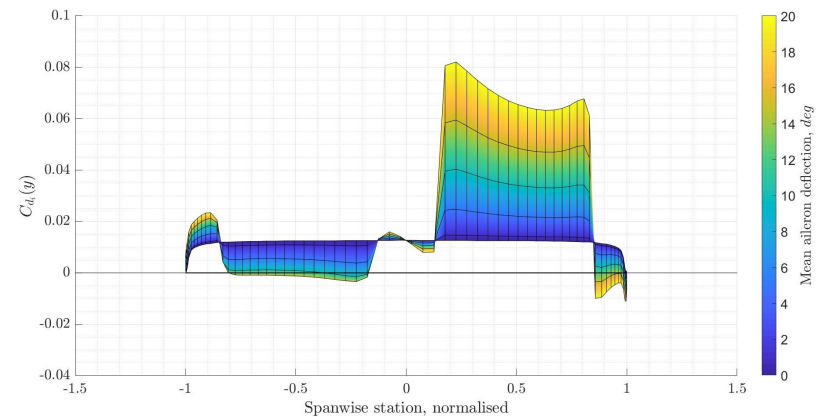

Fig. 5 Elliptic case $C_{d i}(y)$ variation with $\delta_{a}$.

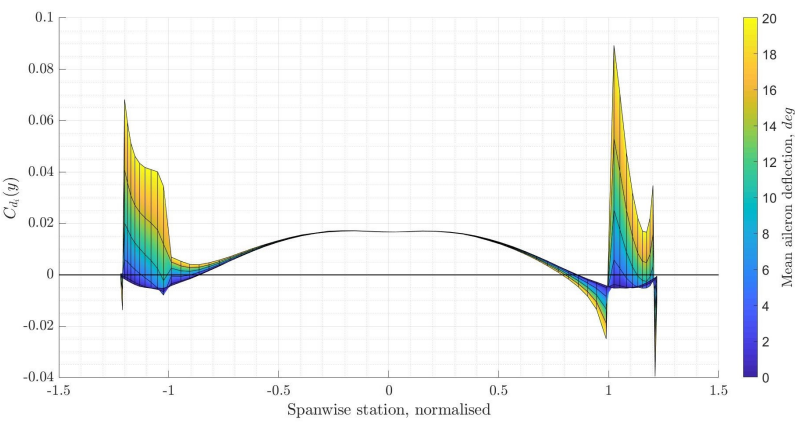

Fig. 6 Prandtl case $C_{d i}(y)$ variation with $\delta_{a}$.

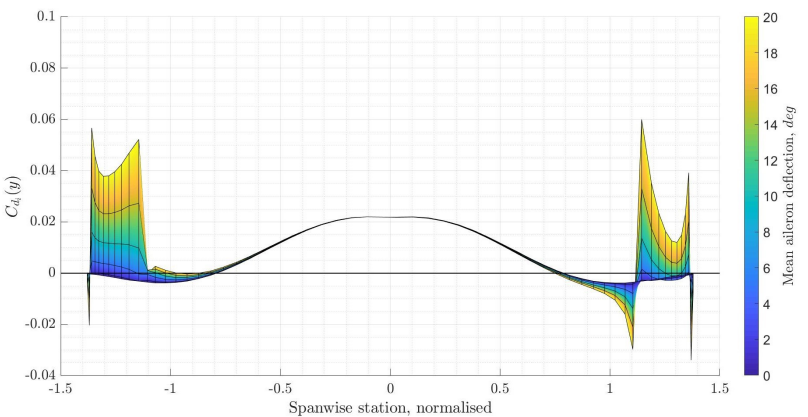

Fig. 7 Cosine case $C_{d i}(y)$ variation with $\delta_{a}$. 

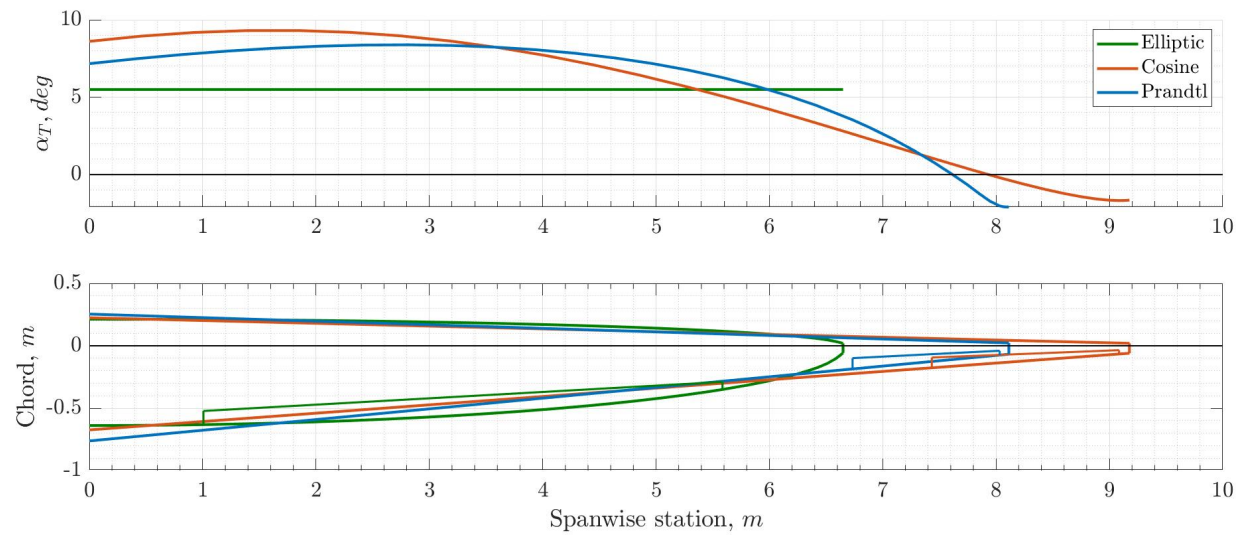

Fig. 8 Comparison of the wing planform, aileron sizing and wing twist distribution for the three configurations.

Table 2 Aileron geometry and stability derivatives for the configurations with maximum proverse yaw.

\begin{tabular}{|l|c|c|c|}
\hline \hline Configuration & Elliptic & Cosine & Prandtl \\
\hline \hline Chord ratio, $r_{f}$ & 0.13 & 0.35 & 0.35 \\
\hline Inboard section ratio, $r_{y 1}$ & 0.15 & 0.81 & 0.99 \\
\hline Outboard section ratio, $r_{y 2}$ & 0.84 & 0.83 & 0.99 \\
\hline Span, $b_{a}(m)$ & 5.71 & 1.65 & 1.30 \\
\hline Surface, $S_{a}\left(m^{2}\right)$ & 0.486 & 0.089 & 0.075 \\
\hline Yawing moment due to aileron deflection, $C_{n_{\delta_{a}}}\left(\mathrm{deg}^{-1}\right)$ & $1.56 \times 10^{-4}$ & $-9.56 \times 10^{-6}$ & $-1.25 \times 10^{-5}$ \\
\hline Rolling moment due to aileron deflection, $C_{l_{\delta_{a}}}\left(\mathrm{deg}^{-1}\right)$ & -0.0065 & -0.0018 & -0.0016 \\
\hline Rolling moment due to roll rate, $C_{l_{p}}\left(\mathrm{rad}^{-1}\right)$ & -0.6407 & -0.6454 & -0.6456 \\
\hline Yawing moment due to rudder deflection, $C_{n_{\delta_{r}}}\left(\mathrm{deg}^{-1}\right)$ & $-9.58 \times 10^{-4}$ & $-7.04 \times 10^{-4}$ & $-7.95 \times 10^{-4}$ \\
\hline Rolling moment due to rudder deflection, $C_{l_{\delta_{r}}}\left(\mathrm{deg}^{-1}\right)$ & $7.20 \times 10^{-5}$ & $5.50 \times 10^{-5}$ & $6.20 \times 10^{-5}$ \\
\hline
\end{tabular}

\section{Experimental Assessment}

The aeroderivatives based analysis carried out in Section III relies on numerous assumptions that simplify and reduce the flight dynamic equations of motion to lateral-directional behaviour. Here, the authors consider the consequent impact of applying the method presented in Section III] to the overall aircraft handling qualities through real-time flight simulations. This is done through the development of $6 \mathrm{DoF}$ nonlinear flight dynamic models for the aforementioned three configurations and their implementation in the engineering flight simulator available at Cranfield University. Handling qualities assessment has been carried out using the two different turning manoeuvres.

\section{A. Nonlinear flight dynamic model}

The nonlinear models retain the full 6 DoF equations of motion that can be found in standard texts such as Cook[22] and Stengel[24]. Aerodynamic forces and moments are also modelled in the first order effects of flight dynamics and control variables. For example, the roll-yaw coupling equations have been modelled as:

$$
\begin{aligned}
& I_{x x} \dot{p}-I_{x z} \dot{r}=L_{v} v+L_{p} p+L_{r} r+L_{\delta_{a}} \delta_{a}+L_{\delta_{r}} \delta_{r} \\
& I_{z z} \dot{r}-I_{x z} \dot{p}=N_{v} v+N_{p} p+N_{r} r+N_{\delta_{a}} \delta_{a}+N_{\delta_{r}} \delta_{r}
\end{aligned}
$$

where the aeroderivatives are scheduled parameters with respect to airspeed and altitude programmed as look-up tables. It should be noted that all tests are carried out within the angle-of-attack and sideslip ranges where the aerodynamic models remain valid and effects such as stall can be neglected. 


\section{B. Engineering flight simulator setup}

The Future Systems Simulator (FSS) in Cranfield University was used for this study. It is a versatile aircraft simulation platform developed to test the integration of various aerospace technologies. Figures 9 and 10 show the cockpit setup and the display setup that acts as the primary human-machine-interface to the pilot. The flight dynamic models developed in MATLAB ${ }^{\circledR} /$ Simulink $^{\circledR}$ environment were deployed on the real-time computer and used in the facility for rapid handling qualities assessments. All flight simulation data was collected at a sampling rate of $50 \mathrm{~Hz}$ together with pilot's control inputs.

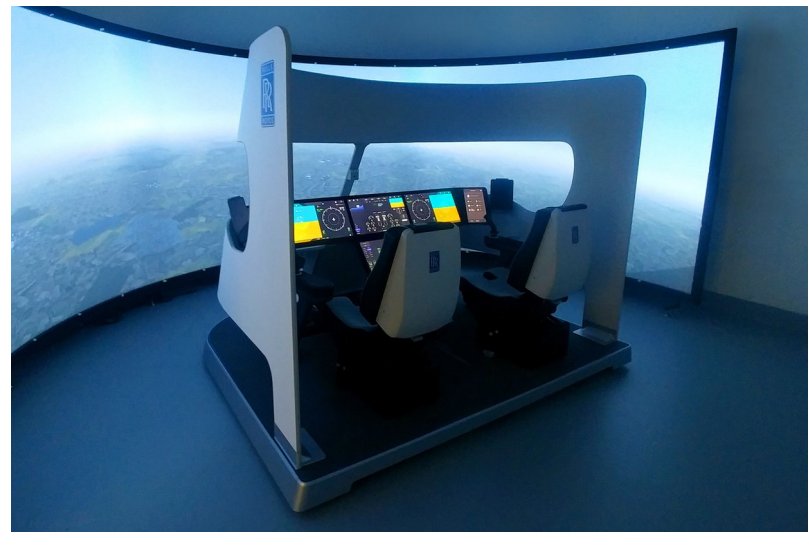

Fig. 9 FSS overall setup.

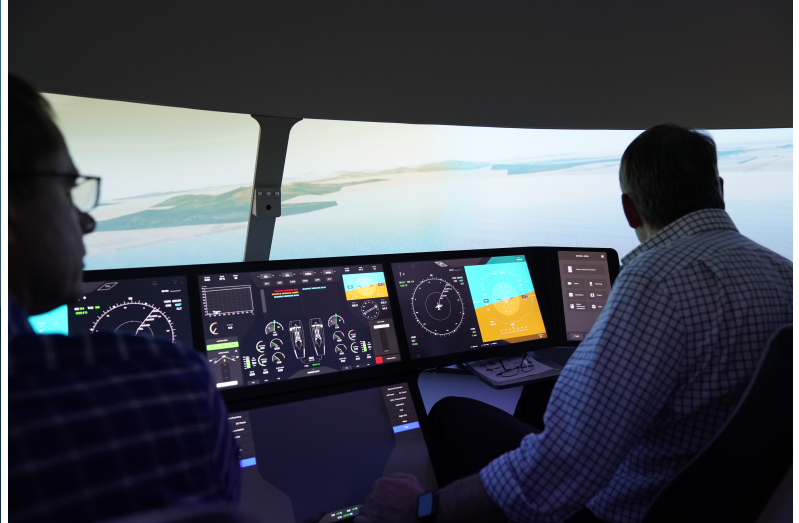

Fig. 10 FSS display setup.

\section{Handling qualities tasks}

The purpose of the flight tests was to assess the lateral-directional handling characteristics of the three configurations. However, the focus lied primarily on the attainment of proverse yaw, and the qualitative and quantitative differences that this would cause during flight. Thus, the tasks were specifically chosen to address such conditions under handling qualitites categories within the Cooper-Harper $(\mathrm{CH})$ rating scale[33], after which the test pilot provided the exact rating. Cooper-Harper rating was made in consideration of the following performance characteristics: Roll control, predictability of roll and yaw response, sidestick and pedal displacements characteristics, and pilot-induced oscillations (PIOs) in roll and yaw axes. The following tasks were carried out:

Bank angle hold: From steady level flight at the design speed of $47 \mathrm{kts}$ and sea level altitude, apply left stick to capture a bank angle of $\phi=\left|20^{\circ}\right|$. Hold for $10 \mathrm{~s}$ and apply right stick to return to steady level flight. Repeat in opposite direction.

Steady turn and heading acquisition: From steady level flight at the design speed of $47 \mathrm{kts}$ and sea level altitude, capture a steady banking turn with $\phi=\left|20^{\circ}\right|$. Maintain bank angle while minimising the resultant sideslip until heading has changed by $\Delta \psi=45^{\circ}$. Repeat in opposite direction.

The suggested Cooper-Harper-Rating criteria were defined as follows:

- Desired performance:

- No PIOs.

- Bank angle capture within $\pm 2^{\circ}$ of target.

- Heading capture within $\pm 2^{\circ}$ of target.

- Adequate performance:

- Bank angle capture within $\pm 5^{\circ}$ of target.

- Heading capture within $\pm 5^{\circ}$ of target.

\section{Pilot Feedback}

Qualitative feedback was provided by the pilot during and after each test. The predominant comment raised throughout the tests was the unstable directional stability of all the configurations, exacerbated by an underdamped Dutch roll mode. 


\section{Task 1}

For the Elliptic configuration, the bank angle capture was found to be difficult, but was attained within $10 s$. However, it was not possible to reach the adequate performance. The main reason for this was that the focus was set on trying to compensate for the heavy sideslip with high rudder inputs due to PIO tendencies. Roll control was therefore sluggish and hard to attain, although the roll rate was found to be fair. The roll mode time constant was found to be very low, which combined with the lightly damped Dutch roll, made the capture and predictability of the task difficult. Overall, the configuration showed major deficiencies in the performance during the task. This required high levels of pilot compensation and was rated a 7 on the Cooper-Harper scale. The Prandtl configuration was found to have fair control under the same setting. Roll response was satisfactory although roll rate was slower even with higher aileron deflections. No PIO tendencies where found and therefore, the predictability and capture of the task was possible. However, capturing a desired bank angle performance required moderate levels of pilot compensation, yielding a rating of 4 on the Cooper-Harper rating scale. The Cosine configuration felt slightly easier to fly than the previous models, but was still rated at 4 . The desired performance was achieved with moderate levels of compensation.

\section{Task 2}

Large demands in sideslip and yaw were observed to attain the heading capture for the three configurations. The Elliptic case exhibited high sideslip angles during the bank that required all the attention of the pilot and withheld any possibility of looking out of the window. The same characteristics as in the first test were observed and thus, the same $\mathrm{CH}$ rating of 7 was given. In the case of the Prandtl configuration, the turn rate built very quickly (overshoot) for a bank of $20^{\circ}$, demanding very rapid roll-out. The predictability was satisfactory, showing no PIOs. The heading capture was adequate but still required corrections from the pilot who gave a $\mathrm{CH}$ rating of 4 . The Cosine model, although still being very loose directionally turned out to be the easiest configuration to execute the task. The roll response was satisfactory and no PIOs were reported. A rating of 4 was provided as the desired performance was still obtained.

Overall, the feature that can be concluded from the above tasks is that the main effort had to be put in minimising sideslip, which did not allow the pilot to focus on any signs of proverse yaw. The Cosine configuration was chosen as the one that provided the most satisfactory handling qualities.

\section{E. Data Analysis}

All the aircraft attitude and the pilot inputs data was collected for quantitative analysis of each test. The data for both tests shows the existence of proverse yaw in the non-elliptic cases. Figure 11 shows the comparison of the lateral-directional performance of each of the configurations acquired during the first task. Proverse yaw can be clearly observed during the roll-in and roll-out of the bank due to aileron input. Here, the yaw rate of the non-elliptic configurations has the same sign as the roll rate, contributing a favourable motion in turn initiation. In contrast, the Elliptic case experiences an opposing yaw rate, as would be expected from adverse yaw. In all cases, rudder was applied with a slight delay from the aileron input. Sideslip is minimised more satisfactorily in the non-elliptic cases, and less oscillations are observed. However, due to the smaller aileron size, roll rates were smaller and more aileron displacement was required. In the same manner, larger rudder displacements were made to compensate for the reduced aileron surface.

The data from the second task is shown in Figure 12 where the same phenomenon is observed. Sideslip was easily kept minimal in the Prandtl and the Cosine configurations, and the new heading was captured with less pilot compensation when compared to the Elliptic configuration. The time to perform the turn had not been specified as part of the performance targets to simplify the task. This criteria might have altered the ratings given to each of the configurations, since the non-elliptic cases were $2 s$ slower than predicted, whereas the Elliptic model attained the new heading within the time but high levels of compensating actions were required to stabilise it at the desired heading.

The root-mean-square (RMS) levels of the aileron and rudder inputs are shown in Table 3 for each test and configuration. The aileron input has been normalised against aileron surface ratio, while the rudder has the same surface in all cases. In these lines, the results show that the aileron to rudder displacements were higher in the non-elliptic cases. Given that these configurations showed more satisfactory handling qualities, the reason for this contrast could have originated in the disparity between aileron size as well as the higher inertia about the yaw axis due to span extension of these configurations. 

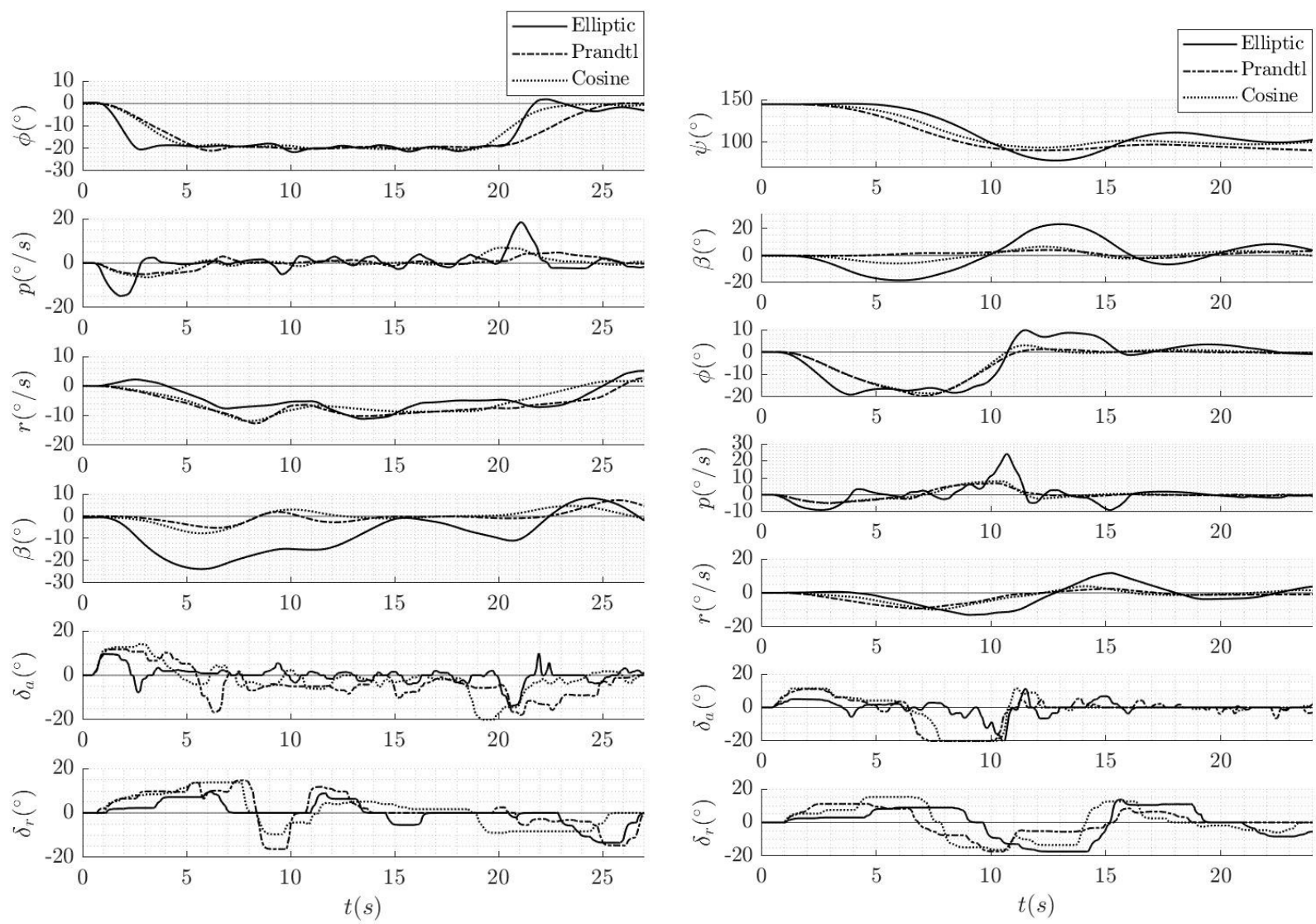

Fig. 11 Flight simulation test data from Task 1.

Fig. 12 Flight simulation test data from Task 2.

Lateral-directional activity was measured according to the metric established in Equation 16 This ratio gives insight into the total pilot activity demanded by each of the tasks given the maximum deflections allowed by the control surfaces.

$$
M_{A}=\frac{1}{N}\left[\frac{\sum_{i=1}^{N}\left|\delta_{a_{i}}\right|}{\delta_{a_{\max }}} \frac{S_{a}}{S_{a_{\text {ref }}}}+\frac{\sum_{i=1}^{N}\left|\delta_{r_{i}}\right|}{\delta_{r_{\max }}}\right]
$$

where $N$ is the total number of data points. The results for this activity metric are shown in Table 4 It is seen that Task 1 exhibits similar activity ratios for all three configurations, while for Task 2 the Elliptic case had the highest activity ratio, followed by the Cosine and the Prandtl. Combining all the aforementioned, it can be concluded that proverse yaw was observed in the flight test data from the Prandtl and Cosine configurations and the pilot feedback reflected improved handling qualities. However, it is difficult to quantify these benefits from the extracted results. Further assessment should be performed after correction of directional stability and modelling of geometrically similar ailerons must be considered for a more reliable comparison.

Table 3 RMS levels for aileron and rudder inputs.

\begin{tabular}{|c|c|c|c|c|}
\cline { 3 - 5 } \multicolumn{2}{c|}{} & Elliptic & Prandtl & Cosine \\
\hline \multirow{2}{*}{ Task 1 } & Aileron RMS & $3.62^{\circ}$ & $1.19^{\circ}$ & $1.28^{\circ}$ \\
\cline { 2 - 5 } & Rudder RMS & $5.15^{\circ}$ & $7.91^{\circ}$ & $7.11^{\circ}$ \\
\hline \multirow{2}{*}{ Task 2 } & Aileron RMS & $4.15^{\circ}$ & $1.31^{\circ}$ & $1.50^{\circ}$ \\
\cline { 2 - 5 } & Rudder RMS & $9.20^{\circ}$ & $7.18^{\circ}$ & $9.67^{\circ}$ \\
\hline
\end{tabular}

Table 4 Ratios for task activity metric $M_{A}$.

\begin{tabular}{|c|c|c|c|}
\cline { 2 - 4 } \multicolumn{1}{c|}{} & Elliptic & Prandtl & Cosine \\
\hline Task 1 & 0.28 & 0.34 & 0.33 \\
\hline Task 2 & 0.50 & 0.31 & 0.44 \\
\hline
\end{tabular}




\section{Discussion of Results}

The analytical approach provided the means to quantify the unconventional behaviour of induced drag during aileron deflection and enable the design of ailerons for proverse yaw. This inverse variation of induced drag observed on the wings with a down-going aileron satisfies the definition of a favourable yawing moment due to aileron deflection caused by induced thrust. Thus, yielding a total drag increase that is smaller than the increment added on a conventional wing with elliptic loading.

The sought effect of the derived aileron designs was observed quantitatively in the simulator test data. Nevertheless, the pilot remained unaware of the adverse yaw correction. In this sense, the pilot did see improved roll and yaw performance in the non-elliptic configurations, but not specifically related to proverse yaw. Furthermore, the rudder to aileron displacements per control surface area were generally higher, even though less pedal activity was required from the pilot to perform the tasks satisfactorily. This fact is contradictory to the expected outcome for a wing designed for proverse yaw performing a coordinated turn, because it is intuitively deduced that a favourable yawing motion should reduce the rudder required to minimise sideslip in a steady turn. However, a linear flight dynamic analysis of this problem shows that this is not the case. Another dominant contributor to this increased rudder displacement could be the presence of directional instability and the underdamped Dutch Roll mode observed in all configurations. This is further exacerbated by the oscillating behaviour of the Elliptic configuration, which further complicates the identification of proverse yaw. Nevertheless, the overall conclusion from the simulator tests agrees with the expected improvement in the lateral-directional handling qualities of the configurations with non-elliptic lift distributions.

A further observation that should be added to the above discussion concerns the assumption of structural equivalence, applied in the form of having equal mass for the three configurations. However, this assumption was not applied in the estimation of the wing inertias. Given that the load is shifted towards the root, an analogous assumption should be made about the distribution of structural weight. This would shift the centroid inboard and therefore the inertias would remain almost the same, which would provide a more reliable comparative study.

Recalling one of the main advantages of these wing designs (mentioned in Section [1], non-elliptic lift distributions could bring favourable lateral-directional capabilities such that there would be no need to have a vertical stabiliser. However, the results presented in this paper do not clarify whether a finless configuration would be able to improve these characteristics. Rudder activity indicates that it would not be possible due to the increased rudder requirement. However, the removal of the fin could yield the consequent elimination of opposing coupling contributions from the fin.

\section{Conclusions}

In this paper the authors have presented a closer inspection of the lateral-directional characteristics of wings with non-elliptic lift distributions that promise further reductions in induced drag when compared to traditional wings designed on the basis of an optimal elliptic lift distribution. This study has focused on removing adverse yaw tendencies and satisfying roll authority requirements and in the process deriving an aileron design method for three wing planforms: (1) a baseline case with elliptic lift distribution, (2) a case with cosine distribution and, (3) a case adhering to the original Prandtl 1933 method. The aileron design spaces containing key geometric parameters were explored for each configuration where only the non-elliptic cases yielded solutions satisfying the proverse yaw and roll authority requirements. These results from the analytical approach were complimented with experimental assessment of the handling qualities through pilot-in-the-loop simulation trials in an engineering flight simulator. The invited test pilot was asked to carry out bank angle hold task and execute a steady turn with heading acquisition and provide qualitative feedback and Cooper-Harper ratings for each configuration.

The use of fundamental low-fidelity methods provided a fast and simple tool to demonstrate a set of solutions that could not have been considered with other traditional approaches (for which an underlying assumption is the optimality of the elliptic spanload for minimum induced drag). However, the assumptions and limitations of this aileron design method were made evident through simulations using the non-linear flight dynamic model. During the piloted trials, proverse yaw was only observed upon turn initiation since only the contribution of aileron deflection to yawing moment was taken into consideration in this design process. The difficulties to analytically capture the nonlinear flight dynamic coupling terms within the aileron design process were exposed further into the manoeuvre, where the dynamics were found to be dominated by lateral-directional coupling effects. 


\section{References}

[1] Duncan, J. S., Pilot's Handbook of Aeronautical Knowledge, $2^{\text {nd }}$ ed., Federal Aviation Administration, Oklahoma City, 2016.

[2] Prandtl, L., "Über tragflügel kleinsten induzierten Widerstandes," Zeitschrift für Flugtecknik und Motorluftschiffahrt, Vol. 24, 1933, pp. 556-561.

[3] Prandtl, L., and Betz, A., "NACA-TR-116: Applications of Modern Hydrodynamics to Aeronautics. [in Two Parts]," Tech. rep., National Advisory Committee for Aeronautics, Goettingen, Germany, 1923.

[4] Nickel, K. L. E., "Minimal drag of wings with prescribed lift, roll moment and yaw moment," Tech. rep., Madison Mathematics Research Center, University of Wisconsin, Madison, 1983.

[5] Phillips, W. F., Alley, N. R., and Goodrich, W. D., "Lifting-Line Analysis of Roll Control and Variable Twist," Journal of Aircraft, Vol. 41, No. 5, 2004, pp. 1169-1176. https://doi.org/10.2514/1.3846

[6] Montgomery, Z. S., Hunsaker, D. F., and Joo, J. J., "A Methodology for Roll Control of Morphing Aircraft," AIAA Scitech 2019 Forum, American Institute of Aeronautics and Astronautics, Reston, Virginia, 2019, p. 18. https://doi.org/10.2514/6.2019-2041

[7] Horten, R., and Selinger, P. F., Nurflügel, Weishaupt, Germany, 1987.

[8] Bowers, A. H., Murillo, O. J., Eslinger, B., and Gelzer, C., "On Wings of the Minimum Induced Drag: Spanload Implications for Aircraft and Birds," Tech. rep., NASA Armstrong Flight Research Center, Edwards, 2016. https://doi.org/NASA/TP-2016219072

[9] Alisport SRL, "Silent 2 Targa Pure,", 1994. URL http://www.alisport.com/?product=silent-2-targa-2

[10] Bragado Aldana, E., and Lone, M. M., “Aerodynamic and flight dynamic study of non-elliptic wing lift distributions," AIAA Scitech 2019 Forum, AIAA, American Institute of Aeronautics and Astronautics, San Diego, California, 2019 , pp. 1-14. https://doi.org/10.2514/6.2019-0820

[11] European Aviation Safety Agency, “CS-22: Certification Specifications for Sailplanes and Powered Sailplanes,” Tech. Rep. Amendment 2, EASA, 2009.

[12] IHS ESDU, "ESDU 88013: Rolling moment derivative , L $\xi$ for plain ailerons at subsonic speeds,” Tech. Rep. August, Royal Aeronautical Society, 1988.

[13] IHS ESDU, “ESDU 88029 - Yawing moment coefficient for plain ailerons at subsonic speeds,” Tech. Rep. November, IHS ESDU, 2006.

[14] Finck, R., and Hoak, D., “USAF Stability and Control DATCOM,” Tech. rep., United States Air Force, United States, 1978.

[15] Toll, T. A., "NACA-TR-868: Summary of lateral-control research," Tech. rep., Langley Aeronautical Lab; National Advisory Committee for Aeronautics, Langley Field, VA, United States, 1947.

[16] Weick, F. E., and Jones, R. T., "NACA-TR-605: Resume and analysis of NACA lateral control research,” Tech. rep., National Advisory Committee for Aeronautics, Langley Aeronautical Laboratory, 1937.

[17] Perkins, C. D., and Hage, R. E., Airplane Performance, Stability and Control, Wiley, University of Michigan, 1949.

[18] Gudmundsson, S., General Aviation Aircraft Design: Applied Methods and Procedures, Elsevier Science, 2013.

[19] Roskam, J., Airplane Flight Dynamics and Automatic Flight Controls, Part 1, DARcorporation, United States, 1998.

[20] Seckel, E., Stability and Control of Airplanes and Helicopters, $1^{\text {st }}$ ed., Elsevier, 1964. https://doi.org/10.1016/C2013-0-07585-8

[21] Roskam, J., Airplane Design Part VII: Determination of Stability, Control and Performance Characteristics, $1^{\text {st }}$ ed., DARcorporation, University of Kansas, 1985.

[22] Cook, M., Flight Dynamics Principles, $3^{\text {rd }}$ ed., Elsevier Ltd., Oxford, 2012.

[23] Glauert, H., "Theoretical Relationships for an Aerofoil with Hinged Flap,” Tech. rep., Aeronatutical Research Committee, London, 1927.

[24] Stengel, R. F., Flight dynamics, $1^{\text {st }}$ ed., Princeton University Press, Princeton, 2004. 
[25] Lekto, W., and Kemp, W. B., "Wind-Tunnel Tests of Ailerons at Various Speeds III - Ailerons of 0.2 airfoil chord and true Contour with 0.35-aileron-chord airfoil," Tech. rep., National Advisory Committee for Aeronautics, Washington, 1947.

[26] Katz, J., and Plotkin, A., Low-Speed Aerodynamics, $2^{\text {nd }}$ ed., Cambridge University Press, Cambridge, 2001. https://doi.org/10. 1017/CBO9780511810329

[27] Anderson, J. D., Fundamentals of aerodynamics, $5^{\text {th }}$ ed., Mcgraw Hill, 2010.

[28] Torenbeek, E., Synthesis of Subsonic Airplane Design, $1^{\text {st }}$ ed., Springer Netherlands, Dordrecht, 1982. https://doi.org/10.1007/ 978-94-017-3202-4.

[29] Howe, D., Aircraft Conceptual Design Synthesis, John Wiley \& Sons, Ltd, Chichester, UK, 2000. https://doi.org/10.1002/ 9781118903094

[30] Sadraey, M. H., Aircraft Design, ${ }^{\text {st }}$ ed., John Wiley \& Sons, Ltd, Chichester, UK, 2012. https://doi.org/10.1002/9781118352700

[31] Raymer, D., Aircraft Design: A Conceptual Approach, Sixth Edition, $6^{\text {th }}$ ed., American Institute of Aeronautics and Astronautics, Inc., Washington, DC, 2018. https://doi.org/10.2514/4.104909.

[32] Drela, M., and Youngren, H., “AVL,”, 2017. URL http://web.mit.edu/drela/Public/web/avl//.

[33] Cooper, G. E., and Harper, R. P., J., "The use of pilot rating in the evaluation of aircraft handling qualities," Tech. rep., NASA Ames Research Center, Washington, 1969. https://doi.org/NASA-TN-D-5153. 
2020-01-05

\section{Closer look at the flight dynamics of wings with non-elliptic lift distributions}

Aldana, Estela Bragado

AIAA

Bragado Aldana E, Mendez A \& Lone M (2020) Closer look at the flight dynamics of wings with non-elliptic lift distributions. In: 2020 AIAA SciTech Forum, 6-10 January 2020, Orlando, Florida https://doi.org/10.2514/6.2020-0284

Downloaded from Cranfield Library Services E-Repository 